\title{
GLAD!
}

Revue sur le langage, le genre, les sexualités

02 | 2017

Varia

\section{Juliette Rennes (éd.). 2016. Encyclopédie critique du genre. Corps, sexualité, rapports sociaux}

\section{Charlotte Thevenet et Julie Abbou}

\section{(2) OpenEdition}

Journals

Édition électronique

URL : http://journals.openedition.org/glad/387

DOI : $10.4000 /$ glad.387

ISSN : 2551-0819

Éditeur

Association GSL

Référence électronique

Charlotte Thevenet et Julie Abbou, « Juliette Rennes (éd.). 2016. Encyclopédie critique du genre. Corps, sexualité, rapports sociaux », GLAD! [En ligne], 02 | 2017, mis en ligne le 01 juin 2017, consulté le 21 janvier 2021. URL : http://journals.openedition.org/glad/387 ; DOl : https://doi.org/10.4000/glad.387

Ce document a été généré automatiquement le 21 janvier 2021.

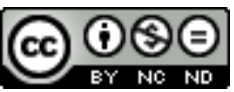

La revue GLAD! est mise à disposition selon les termes de la Licence Creative Commons Attribution Pas d'Utilisation Commerciale - Pas de Modification 4.0 International. 


\title{
Juliette Rennes (éd.). 2016. Encyclopédie critique du genre. Corps, sexualité, rapports sociaux
}

\author{
Charlotte Thevenet et Julie Abbou
}

\section{RÉFÉRENCE}

Juliette Rennes (éd.). 2016. Encyclopédie critique du genre. Corps, sexualité, rapports sociaux, Paris : Éditions La Découverte. 752 pages.

1 Les années 2000-2010 ont vu le développement institutionnel en France et le déploiement dans les sciences humaines des études sur le genre et les sexualités (créations de masters, d'associations, de revues) dont GLAD! prenait acte en introduction à son premier numéro. C'est également de ce constat que part l'Encyclopédie critique du genre, éditée par Juliette Rennes et publiée cet hiver (2016) aux Éditions La Découverte. Face à «la présence limitée, au sein des dictionnaires et des ouvrages de synthèse francophones sur le genre parus dans les décennies 1990-2000, d'une très grande partie des domaines couverts par cette Encyclopédie critique du genre» (p. 25), l'ouvrage vient combler un vide éditorial francophone. "État des savoirs » (p. 22) mais aussi configuration critique d'un champ invitant à renouveler ses questions de recherche, l'ouvrage nourrit sa dimension encyclopédique d'un positionnement constructiviste fort visant à dénaturaliser l'évidence des rapports sociaux. La structure même de l'ouvrage reflète cette dimension critique. L'encyclopédie et ses entrées sont assorties d'un riche paratexte, qui témoigne du souci des auteur.es de fournir une réflexion épistémologique. 


\section{Une approche encyclopédique pour décrire et analyser "la chair des rapports sociaux »}

2 Ainsi que l'expliquent les auteur.es de l'introduction appelée «La chair des rapports sociaux " (Juliette Rennes, Catherine Achin, Armelle Andro, Laure Bereni, Alexandre Jaunait, Luca Greco, Rose-Marie Lagrave, Gianfranco Rebucini), l'ouvrage s'est élaboré autour de cette invitation à " contribuer collectivement [...] à décrire et analyser [...] la "chair des rapports sociaux" " (p. 24) : chair de l'empirique, mais aussi compte rendu des "asymétries structurelles" (p. 24) dans les interactions, et enfin "épaisseur historique » (p. 25) tant des objets que des approches, qui permet d'allier tout au long de l'ouvrage analyses de cas et réflexion épistémologique.

3 L'ouvrage s'organise autour de trois axes : le corps, la sexualité et les rapports sociaux. Se positionnant « contre la caricature conservatrice d'une "théorie du genre" qui serait un simple mot d'ordre de contestation in abstracto des rôles traditionnellement féminins et masculins » (p. 24), les entrées font une place de choix à la complexité des "formes d'incorporation du genre» (p. 13): on y trouve des entrées faisant écho aux critères discriminant et classant les corps (" Âge ", « Beauté ", « Handicap », « Poids ", "Taille", "Corps légitime», "Vêtement»), d'autres renvoyant davantage à des pratiques («Sport», «Désir(s)», «Danse»), d'autres enfin s'attachent à désessentialiser le corps dans ses manifestations apparemment les plus naturelles: «Fluides corporels», mais aussi reproduction («Bioéthique et techniques de reproduction »), «Organes sexuels », «Puberté », "Virginité » ou encore "Voix ». Le corps y est toujours considéré comme un objet construit, auquel ne donne sens que la multiplicité des rapports dans lesquels il est pris, comme le montrent les trois notices faisant apparaître le mot «corps » : «Corps au travail », «Corps légitime » et «Corps maternel ».

4 La sexualité constitue le deuxième grand axe ayant guidé l'élaboration du volume, et ici encore, on peut saluer la diversité d'approches que permettent les différentes entrées: la «Santé » («VIH/sida » bien sûr, mais aussi «Gynécologie »), le plaisir (« Plaisir sexuel »), l'éducation («Éducation sexuelle»), le travail («Prostitution», « Pornographie »)...

5 Le troisième axe, "l'approche multidimensionnelle des rapports sociaux ", cherche à éclairer « l'importance que revêt désormais, dans les travaux sur le genre, l'articulation des rapports de genre avec d'autres rapports sociaux» (p.20). "[C]ette approche "intersectionnelle", “imbriquée", "multidimensionnelle" ou "consubstantielle" " (p. 20) des rapports sociaux trame ainsi l'Encyclopédie, comme en témoigne la présence des entrées "Handicap ", " Mondialisation », "Postcolonialités ", " Queer », " Race », "Religion", "Trans' ", notamment. On pourrait remarquer que cet axe semble davantage une préoccupation épistémologique donnant sa consistance à l'ouvrage, qu'un thème à proprement parler. Ainsi présenté, il renvoie effectivement le corps et la sexualité à des thématiques différenciées, alors même que l'Encyclopédie critique tend précisément à n'aborder corps et sexualités que dans l'épaisseur des rapports sociaux. Mais c'est peut-être là l'écueil de la linéarité du langage, à laquelle même un format encyclopédique ne permet pas d'échapper totalement, nécessitant de saisir ou, du moins, de nommer les rapports sociaux et les constructions sociales, tout imbriqués soient-ils, les uns après les autres. Ces axes dessinent alors peut-être davantage les principes qui ont guidé ce travail que les notices qui en résultent, et à ce titre, en 
éclairent la lecture. Pour poursuivre ce projet encyclopédique, les auteur.es ont également fait le choix de l'hétérogénéité et de la posture critique et située.

\section{Une encyclopédie ouverte}

6 Loin de prétendre à un état des lieux exhaustif, c'est en effet par l'ouverture que se caractérise cette Encyclopédie critique du genre. Tout d'abord, le sommaire des différentes entrées, loin de tenter de forcer une unité de façade, révèle le pari (réussi) de la diversité qu'ont fait les auteur.es. À côté des entrées disciplinaires, comme "Langage ", "Arts visuels » ou "Psychanalyse », on trouve également des notices aux titres plus inattendus telles que «Objets». Les concepts traditionnels du genre («Bicatégorisation», «Incorporation») cohabitent avec des notions plus larges («Espace urbain» ou «Nation»). Ce foisonnement des catégories va de pair avec la pluridisciplinarité annoncée en introduction. Non seulement l'Encyclopédie présente-telle un éventail de quinze disciplines, mais encore chaque auteur.e a-t-iel été encouragé.e à ne pas se cantonner à sa propre discipline dans l'écriture de sa notice.

7 Cette ouverture disciplinaire fait écho à une ouverture formelle: les entrées sont reliées à un index permettant de naviguer dans l'ouvrage de manière transversale. L'index constitue ainsi une agréable porte dérobée à l'Encyclopédie pour en faire une lecture buissonnière.

8 Enfin, l'Encyclopédie se veut ouverte sur «les enjeux politiques des domaines et objets traités» (p.23), c'est sa dimension critique, affirmée à travers une "perspective intersectionnelle» (p. 21). Ouvert sur les rapports sociaux auxquels les études de genre ont pu parfois se montrer aveugles, l'ouvrage affirme la nécessité épistémologique et politique d'une approche intersectionnelle.

9 À la lecture, cette diversité conceptuelle, d'objets, d'approches et de forme fonctionne bien comme une ouverture et non un éparpillement, qui permet de renouer avec la dimension exploratoire et en devenir du genre encyclopédique.

\section{Une encyclopédie critique}

10 L'Encyclopédie se caractérise également par sa volonté critique. Les auteur.es, tout en prenant soin de fournir un état des savoirs, «ne s'interdisent pas d'adopter une position et ne s'expriment pas d'une seule voix» (p.24). Pas de prétention à l'objectivité, ce vieux serpent de mer académique, dans l'Encyclopédie, mais plutôt des notices offrant un état des lieux critique, c'est-à-dire engagé et présenté comme tel. Ainsi, par exemple, de la notice «Pornographie» dont les auteurs, Mathieu Trachman et Florian Vörös, annoncent vouloir «montrer [...] que le refus de constituer la pornographie en symbole de l'oppression des femmes ne conduit pas à abandonner le projet d'une analyse féministe de la pornographie» (p.479): une prise de position " pro-sexe » explicite qui n'entrave évidemment pas la visée scientifique de l'article.

11 Dans le même sens, le choix des notices relève d'un positionnement critique héritier des pratiques en études de genre: celui d'un joyeux constructivisme. Cela se révèle dans l'absence de notice "Sexe» au profit d'entrées "Organes sexuels » et « Mâle/ femelle» entre autres. Pas de notice "Viol " mais une entrée "Violence sexuelle» favorisant, malgré son singulier, la prise en compte de tout le spectre des violences 
sexuelles et surtout des dénominations sur lequel l'auteure, Alice Debauche, insiste. Plutôt qu'une seule dénomination, ce sont parfois des couples de concepts qui sont privilégiés : «Inné/acquis », "Hétéro/homo » (et "Bicatégorisation») mettant immédiatement en évidence la co-constitution de ces phénomènes.

\section{Une encyclopédie située?}

Enfin, l'Encyclopédie critique du genre a l'ambition d'une ouverture géographique et linguistique, qui dépasse "la mention de références bibliographiques étatsuniennes " pour présenter "des cas, des recherches et des débats issus de diverses aires géographiques » (p.23). Qu'il s'agisse des «cultures mélanésiennes » des «HautesTerres de Papouasie-Nouvelle-Guinée » abordées dans l'entrée « Fluides corporels » (coécrite par Nahema Hanafi et Caroline Polle) ou du «berdache amérindien » (abordé dans «Hétéro/homo» de Sébastien Chauvin et Arnaud Lerch), les notices ne se concentrent pas sur la France, ni sur les États-Unis habituellement surreprésentés en études de genre, mais s'ouvrent mondialement.

Cependant, cette volonté d'éviter les autoroutes du savoir afin d'élargir le champ des études de genre aux travaux existants ne parvient pas tout à fait à son but, et l'Encyclopédie reproduit les travers qu'elle pointe, avec un corpus scientifique majoritairement en anglais américain. Deux entrées, "Consommation» écrite par Leora Auslander et «Psychanalyse » de Adrienne Harris et Eyal Rozmarin, sont traduites, manifestement de l'anglais mais sans que cela ne soit précisé; seuls apparaissent les noms des traducteur.ices (respectivement Christophe Jaquet, et Alexandre Jaunait, Marie-Nadine Prager et Idan Segev). Ce choix, dans un ouvrage francophone, aurait mérité d'être explicité ; et surtout, la langue d'écriture de ces articles d'apparaître, dans un geste invitant à déconstruire l'évidence de l'anglais étatsunien comme lingua franca et comme langue hégémonique. Mentionner systématiquement les traducteur.ices dans les références bibliographiques de fin de notice aurait également permis de reconnaître et de visibiliser le travail de ces passeur.es qui ne cessent de nourrir le champ francophone des études de genre.

Cette présence de l'anglais est bien sûr difficilement évitable en études de genre, d'un point de vue historique comme linguistique. Les écarts de réception et les mouvements de traduction entre mondes anglophone et francophone ont façonné l'histoire des études de genre francophones et eu une influence sur nombre de débats théoriques. Mais plutôt que de tenter une échappée, il aurait peut-être été plus fructueux de reconnaître cette influence tout en tentant de la nuancer. L'insertion d'une notice «Traduction» par exemple aurait permis de réfléchir aux échanges entre les aires états-unienne et francophone dans l'histoire des études de genre, échanges qui ont donné lieu à des transformations et réinventions, tant une traduction ne se réduit jamais à un simple transfert. Comment, en effet «élaborer des enquêtes et de [...] nouvelles questions de recherche [...] enrichies par ce regard collectif sur un certain moment des études de genre » (p. 25) si on ignore de quel champ linguistique on parle? Dans la mesure où l'Encyclopédie non seulement revendique son "ouverture internationale", mais encore se place dans un vide éditorial francophone, on regrettera donc que l'ouvrage ne problématise pas davantage sa fonction d'ouvrage passeur. 

la forme d'une «note sur le genre grammatical » (p. 11) qu'une recension dans GLAD! pouvait manquer de repérer. Les éditeur.ices de l'Encyclopédie ont « souhaité adopter un langage non sexiste » (p.11) et «tendre vers un langage mixte» (p. 11) ; loin d'unifier l'écriture de l'ouvrage en préférant une forme aux autres, les éditeur.ices ont laissé les auteur.es libres d'utiliser « les ressources diverses » (p. 11) de l'écriture inclusive : point médian, formes épicènes, formes inclusives. Cette position critique vis-à-vis du langage, qui ne remplace pas une forme prescriptive par une autre mais embrasse et encourage la diversité des formes de l'écriture inclusive dans leur créativité, relève d'une conception de la langue vivante et en constante transformation, fidèle à l'ouverture qui caractérise l'ouvrage.

17 Ainsi, dans le format encyclopédique, dans les éléments qui le composent formellement, dans le choix des entrées ainsi que dans la rédaction des notices transparaît une approche du genre qui soutient son ambition théorique par une méthode et une épistémologie de la complexité, et qui fait de cet ouvrage un apport indiscutable au champ. La vitalité, l'ambition et la nuance critique dont témoigne cette Encyclopédie critique du genre en feront également, sans aucun doute, un outil précieux à tout.e chercheur.e en études de genre désireux.se d'explorer des aspects du champ qui ne lui sont pas familiers, ou tout simplement de s'ouvrir à d'autres approches et d'autres disciplines.

\section{INDEX}

Thèmes : Actualités

Mots-clés : intersectionnalité, rapports sociaux, pluridisciplinarité

Keywords : intersectionality, social relations, pluridisciplinarity 